\title{
Sequels after implantation of intraocular collamer lens
}

\begin{abstract}
Purpose: To report a case complicated with culture-positive bacterial endophthalmitis and retinal detachment following implantation of a Staar intraocular Collamer lens (ICL) of the right eye.

Methods: The patient presented 2 weeks after uneventful sutureless surgery with decreased visual acuity, redness, and photosensitivity and hypopyon. Prompted pars plana vitrectomy (PPV) and intravitreal injection of antibiotic agents. Vitreous culture was positive for coagulase-negative Staphylococcus epidermidis. Three months later, he presented with blurry vision and the uncorrected visual acuity (UCVA) was 20/200; retinal detachment (RD) was observed. RD was managed surgically.

Result: At the most recent visit, the UCVA was 20/30 with stable ICL and quiet eye.

Conclusion: Bacterial endophthalmitis and retinal detachment are a potential complication of ICL implantation. A proper treatment of postoperative complications is the key role in saving vision. Preoperative counseling of postoperative complications is an important aspect before any intraocular procedure.
\end{abstract}

Keywords: ICL implantation, endophthalmitis, retinal detachment, pars plana vitrectomy
Volume 2 Issue 4 - 2015

\author{
Ali MAl Halafi \\ Department of Surgery, Security forces Hospital, Saudi Arabia
}

Correspondence: Ali M Al Halafi, Consultant and Vitreoretinal Surgeon, Department of Surgery, Ophthalmology Division, Security forces Hospital, PO Box 3643, Riyadh II 48I, Saudi Arabia, Tel +966-I-8024444, Fax 00 +966-I-476-4757, Emailamm-ry@hotmail.com

Received: April 19, 2015 | Published: May 08, 2015
Abbreviations: ICL, intraocular collamer lens; PPV, pars plana vitrectomy; UCVA, uncorrected visual acuity; RD, retinal detachment; PIOLs, phakic intraocular lenses; US FDA, united states food and drug administration

\section{Introduction}

Phakic intraocular lenses (PIOLs) are an emerging procedure within the field of refractive surgery and accepted as an alternative procedure for ametropia correction among various refractive techniques. The Visian Implantable Collamer Lens (ICL; STAAR Surgical Co, Monrovia, California) is the only posterior PIOL that currently approved by the United States Food and Drug Administration (US FDA) for the treatment of moderate to severe myopia. ${ }^{1}$

Endophthalmitis is characterized by marked inflammation of intraocular fluids and tissues. The major category is acute-onset, postoperative endophthalmitis, which is defined as endophthalmitis presenting within 6 weeks of intraocular surgery. Chronic postoperative endophthalmitis presents more than 6 weeks following intraocular surgery. In acute-onset postoperative bacterial endophthalmitis, there is typically acute-onset visual loss and redness, associated with marked intraocular inflammation, anterior chamber, fibrin, and hypopyon. ${ }^{2}$

\section{Case report}

A 22-year-old male with a history of myopic astigmatism presented for evaluation for surgical refractive correction. The cycloplegic refraction was $-8.50-2.75 \times 25$ with corrected visual acuity (VA) $20 / 22.5$ in the right eye and -3.25-3.50x155 2with (VA) 20/20 in the left eye. Corneal findings consistent with corneal thinning according to corneal topography. Fundus examination was unremarkable. The patient underwent toric ICL. A Visian toric phakic ICL was selected for the right eye because of the degree of myopic astigmatism correction and laser epithelial keratomileusis) (LASEK) surgery was planned for the left eye later. It was implanted uneventfully in a center operating room certified for outpatient cataract surgery. The surgery was performed using a sutureless technique following a topical anesthetic eye drops. Povidone-iodine 5\% solution was used to sterilize the eye before the procedure. Moxifloxacin eye drops 4 times per day and prednisone $1 \%$ eye drops 4 times per day were started on the day of surgery. The early postoperative recovery was acceptable; the UCVA was 20/25 on the first postoperative day. After 2 weeks, while still on the antibiotic course, the patient presented with complaints of sudden onset of blurry vision, with severe pain, redness and photosensitivity in the right eye. The UCVA was hand motion, and there were anterior chamber cells and hypopyon and the ICL was well-positioned (Figure 1).

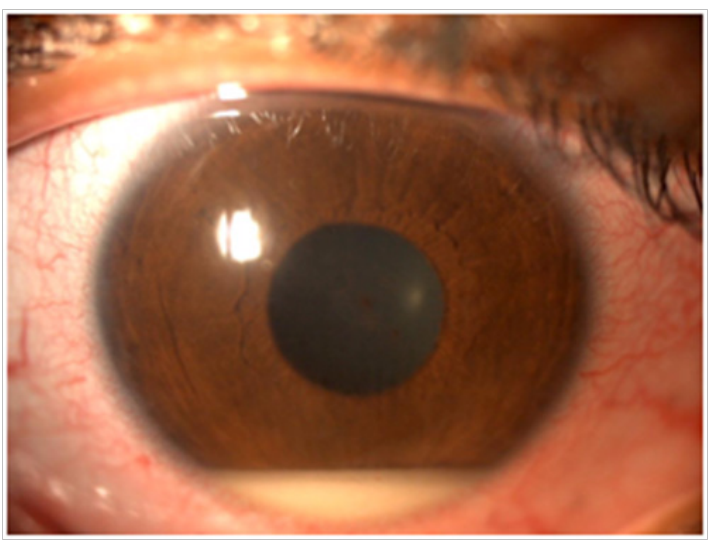

Figure I Slit lamp photograph of hypopyon.

The patient was immediately referred to a vitreoretinal consultant, who noted the vitreous was haze. B-scan ultrasound showed vitreous cells without retinal detachment. Probable acute bacterial endophthalmitis was diagnosed. Twenty-three gauge-three port Pars plana vitrectomy (PPV) system was performed. Aqueous and 
vitreous samples were taken before opening the infusion cannula. Core vitrectomy was done. Intravitreal injection of vancomycin and ceftazidime in standard doses was injected at the end of PPV procedure. Aqueous and vitreous samples were sent for Gram stain, aerobic and anaerobic cultures. Gram stain of the aqueous revealed a few white blood cells showed no organisms. Aqueous cultures were negative. Vitreous Gram stain showed white blood cells and gram-positive cocci, and vitreous cultures grew coagulase-negative Staphylococcus epidermidis. The symptoms and inflammation improved rapidly over the next several days. The gatifloxacin eye drops were continued for 2 weeks, and the prednisolone eye drops were tapered over the following month. One month after PPV, the UCVA was 20/25. Three Months later, he presented to the clinic complaining of blurred vision of the same eye. His vision was hand motion of the right eye. Anterior segment examination was unremarkable with ICL in place. Fundus exam showed retinal detachment involving the macula with super temporal break. The patient was admitted for PPV, endolaser and gas injection (C3F8). Postoperatively, there was no anterior segment inflammation and the retina was flat under the gas (Figure 2) (Figure 3 ). At the most recent visit, the UCVA was 20/30 with stable ICL and quiet eye.

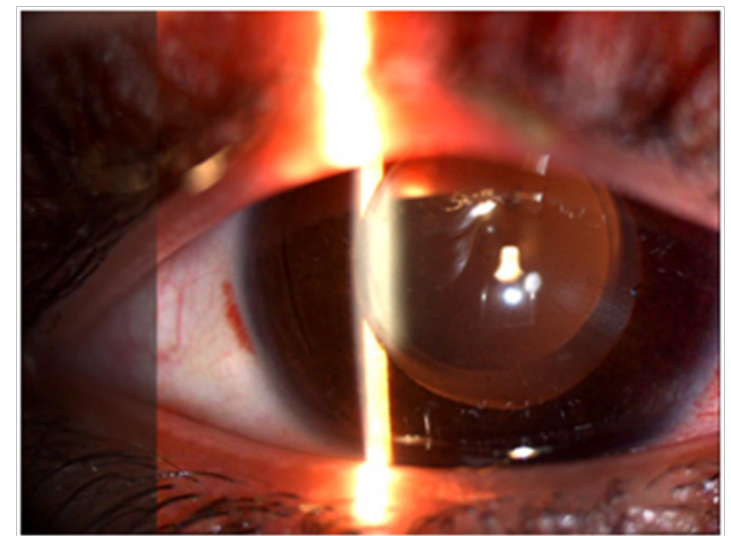

Figure 2 Slit lamp photograph of well-centered ICL after PPV for endophthalmitis.

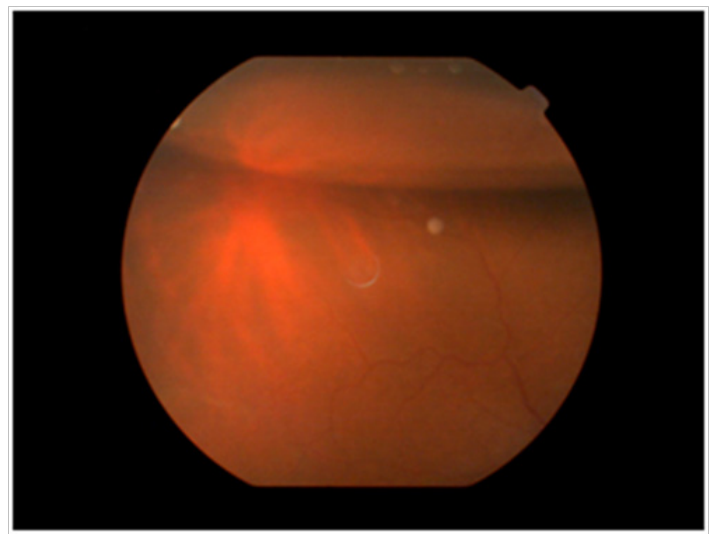

Figure 3 Fundus photograph showing flat retina and the remaining gas after PPV for retinal detachment.

\section{Discussion}

ICL is an alternative to corneal refractive procedures, which preserve accommodation and are limited by corneal thickness. An increased risk for anterior and posterior segment complications was reported. Infective endophthalmitis is a clinical diagnosis but may be confirmed by evaluation of intraocular fluid specimens. The clinical signs are variable and depend on the infecting organism, the duration of infection, the associated inflammation, and various patient risk factors, such as prior surgery, trauma, and immune status.

Implantation of a posterior chamber PIOL carries a potential risk for intraocular complications such as endophthalmitis and retinal detachment. Allan et al. ${ }^{3}$ conducted an online survey of 234 surgeons in 21 countries to show how many of their ICL cases had been complicated by endophthalmitis between January 1998 and December 2006. During the study period, 3 surgeons reported 1 case of endophthalmitis each, a rate of $0.0167 \%$ or approximately 1 case of endophthalmitis per 6000 ICL implantations. A case of culturepositive bacterial endophthalmitis 4days following ICL implantation was reported with full visual recovery after proper treatment. ${ }^{4}$

High myopia and long axial length are risk of retinal detachment after ICL implantations. Retinal detachments were reported in 3 of 526 eye sin the US FDA trial..$^{5}$ In a study consist of 61 eyes, retinal detachment was reported in 1 eye 15 months after ICL implantation which was thought to be secondary to the pre-existing axial length of $31.0 \mathrm{~mm}$ in this case. ${ }^{6}$ Retinal detachment was reported in 11 of 628 eyes implanted with the ICL V4, Martinez-Castillo et al. ${ }^{7}$

\section{Conclusion}

The risk for permanent visual loss associated with intraocular surgery is an important aspect of preoperative counseling before any intraocular procedure. Risk estimates are based on a variety of possible causes for visual loss, but intraocular infection rates are a key consideration. A proper treatment of postoperative complications is the key role in saving vision.

\section{Acknowledgments}

None.

\section{Conflicts of interest}

Authors declare that there is no conflict of interest.

\section{References}

1. Lovisolo CF, Reinstein DZ. Phakic intraocular lenses. Surv Ophthalmol. 2005;50(6):549-587.

2. DoftB.Endophthalmitis Management. Ophthalmology. 1997;104:390-396

3. Allan BD, Argeles-Sabate I, Mamalis N. Endophthalmitis rates after implantation of the intraocular collamer lens: survey of users between 1998 and 2006. J Cataract Refract Surg. 2009;35(4):766-769.

4. Davis MJ, Epstein RJ, Dennis RF, et al. Culture-positive endophthalmitis after implantation of intraocular collamer lens. J Cataract Refract Surg. 2009;35(10):1826-1828

5. Sanders DR, Doney K, Poco M. United States Food and Drug Administration clinical trial of the Implantable Collamer Lens (ICL) for moderate to high myopia: three-year follow-up. Ophthalmology. 2004;111(9):1683-1692.

6. Chang JS, Meau AY. Visian Collamer phakic intraocular lens in high myopic asian eyes. J Refract Surg. 2007;23(1):17-25.

7. Martinez-Castillo V, Boixadera A, Verdugo A, et al. Rhegmatogenous retinal detachment in phakic eyes after posterior chamber phakic intraocular lens implantation for severe myopia. Ophthalmology. 2005;112(4):580-585. 